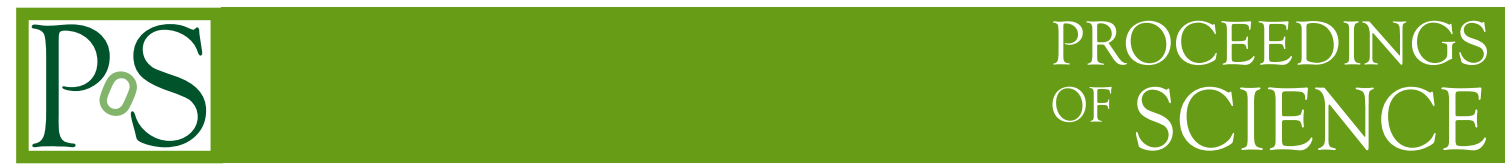

\title{
Investigation of parton densities at very high $\mathrm{x}$
}

\author{
R. Aggarwal*i \\ (Savitribai Phule Pune University) \\ On behalf of the ZEUS Collaboration \\ E-mail: ritu.aggarwallegmail.com
}

The knowledge of the proton parton densities for large $\mathrm{x}$ is very important in the search for new physics signals at the LHC. For Bjorken $x$ larger than 0.6 they are however poorly constrained by the data used in extracting the proton parton density functions (PDFs) and different pdf sets have large uncertainties, and differ considerably, in this regime. We compare the pdf sets most widely used by the LHC community to the ZEUS high $\mathrm{x}$ data. This data has not been previously used in PDF set determinations. Due to the small expected and observed numbers of events in this kinematic regime, Poisson statistics is used in the evaluation of the probabilties assigned to the different PDF sets. A wide variation is found in the ability of the PDF sets to predict the observed results.

European Physical Society Conference on High Energy Physics - EPS-HEP2019 -

10-17 July, 2019

Ghent, Belgium

\footnotetext{
*Speaker.

$\dagger$ Supported by Dept. of Science and Tech. (DST), India.
} 


\section{Introduction}

The knowledge of the distribution of partons inside proton is of utmost importance for analysing the final state in the collisions of protons (or anti-protons). Electron proton Deep Inelastic Scattering (DIS) data collected by the ZEUS and H1 experiments at HERA are the main source for understanding parton distribution functions (PDFs) inside proton. However the Neutral current (NC) ep cross section are measured upto a value of Bjorken $\mathrm{x}$ of 0.65 [1]. Beyond this limit, the PDFs mainly rely on the knowledge gained from fixed target experiments $[2,3,4,5,6]$ which have a disadvantage of nuclear effects. The NC high $\mathrm{x}$ DIS cross sections [7] measured by the ZEUS detector are the only DIS data that cover Bjorken x range upto a value of 1. Although this data has not been included in the theoretical calculation of PDFs, this data tend to contain valuable information to constrain the PDFs in a better way at the highest values of $\mathrm{x}$, where they at present have large uncertainties.
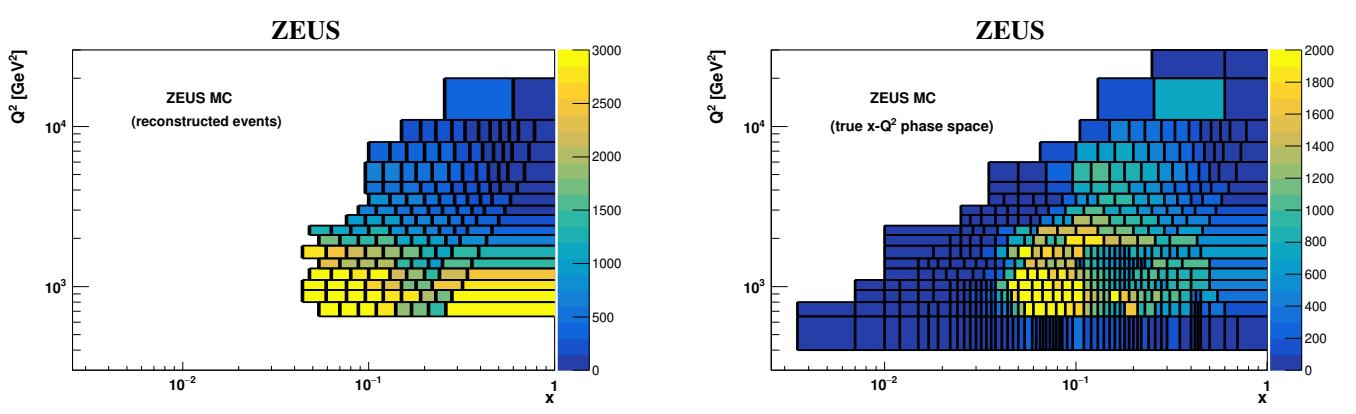

Figure 1: Distribution of number of events in the simulated data after all analysis selections are applied, shown as functions of the (Left:) reconstructed kinematic quantities in cross section bins used in [7], (Right:) generated kinematic quantities in the extended binning scheme.

\section{Transfer Matrix for high-x data}

One of the reasons to why the data could not be included is that some of the bins where crosssections are reported have very small number of events expected and reconstructed. And therefore they have to be dealt using Poisson statistics. In this section the method to develop a Transfer Matrix for the ZEUS high $\mathrm{x}$ data is briefly discussed. Using the Transfer Matrix one can predict the number of events expected in the given cross sections bins (vector $v$ ) from any modern PDF using the Born-level integrated cross sections. Figure 1(a) shows the distribution of reconstructed events in the phase space of reconstructed variables (Bjorken $\mathrm{x}$ and $Q^{2}$ ). The true distribution of these events is somewhat different and is shown in Figure 1(b). Figure 1(b) also shows the finer and extended binning used to map the full true phase space (at the generated level). The Transfer Matrix will map the way events migrate from the generated level (as shown in Figure 1(b)) to the co-ordinates or bins at the reconstructed level (as shown in Figure 1(a)). Each element in the Transfer Matrix is thus given as

$$
t_{i j}=\frac{\sum_{m=1}^{M_{i}} \omega_{m} I(m \in j)}{\sum_{m=1}^{M_{i}} \omega_{m}^{M C}},
$$



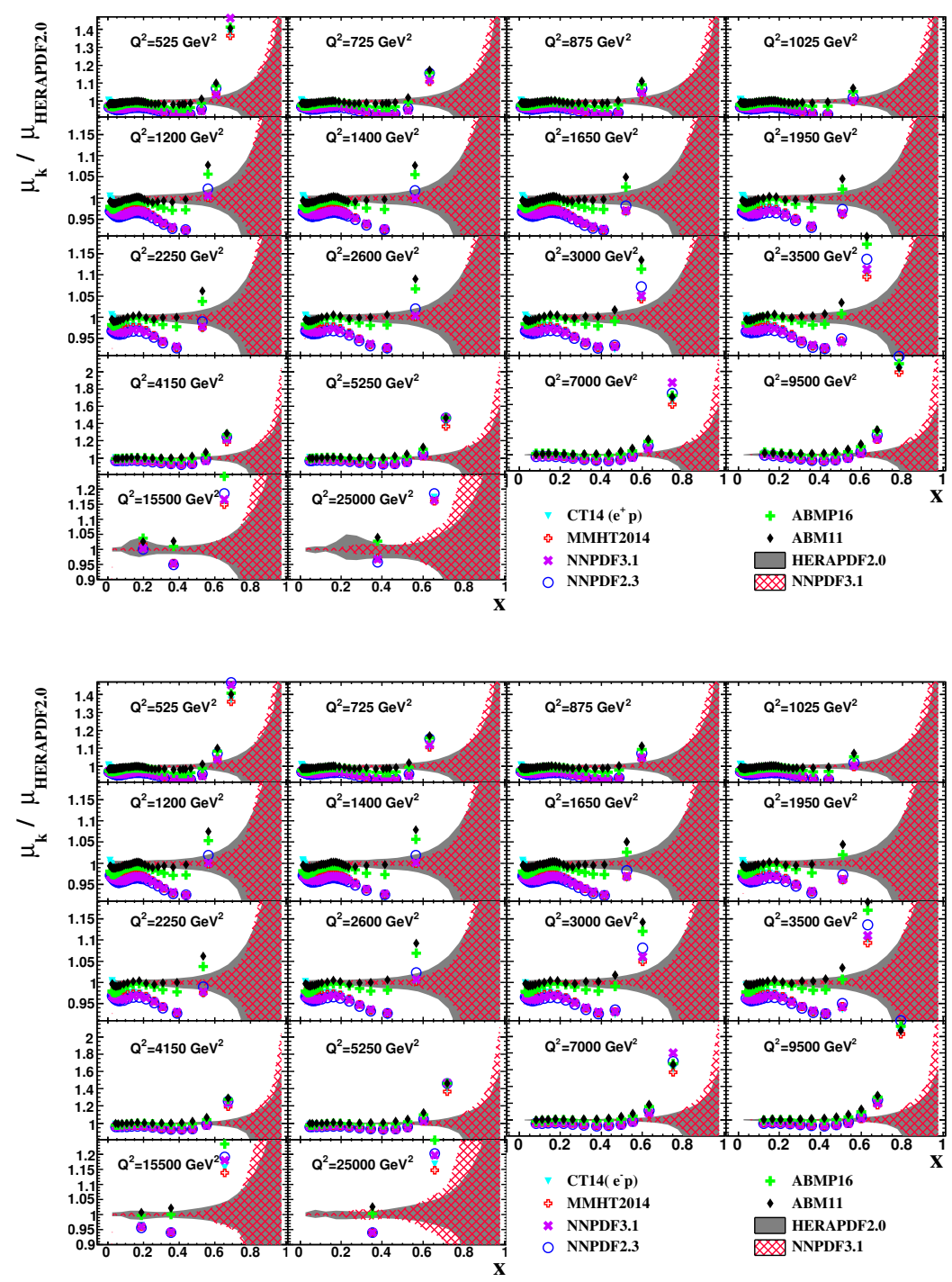

Figure 2: The ratio of the number of generated events from different PDFs to HERAPDF2.0 for $\mathrm{e}^{+} \mathrm{p}$ (top) and $\mathrm{e}^{-} \mathrm{p}$ (bottom) data respectively. The PDF uncertainty on the cross section at the generated level is shown in filled area for HERAPDF2.0 and cross hatched area for NNPDF3.1.

Where $M_{i}$ is the total number of Monte Carlo events generated in bin $i$ defined using the exchanged photon information and $\omega_{m}$ the total weight given to the $m^{\text {th }}$ event, which is product of events weights, $\omega_{m}^{M C}$ and $\omega_{m}^{s i m}$. Here $\omega_{m}^{M C}$ is the generated event weight and $\omega_{m}^{\text {sim }}$ contains weight due to detector's limited acceptance and efficiencies. Indicator function, $I=1$ if event $m$ is reconstructed in bin $j$, else $I=0$.

The expecation for the number of events in the cross sections bins (here referred as vector $\mathrm{N}$ ), is given as

$$
N=T \mu,
$$

where $\mathrm{T}$ is the Transfer Matrix whose each element is calculated for the simulated data using Eq. 2.1 
and $\mu$ is the vector containing events at the generator level in the extended bins.

The events were generated using HERACELES [8], which applies QED and QCD corrections to the Born level cross-sections. The events at the generator level can be obtained from the Born level integrated cross sections (given by vector $\lambda$ ) as shown in the equation given below.

$$
\mu=L R \lambda,
$$

where R matrix incorporates all the corrections done by the HERACELES. The non-diagonal elements of the matrix $\mathrm{R}$ are taken to be zero and it is verified that it is appropriate for our case. Here $L$ is the luminosity of the high $x$ ZEUS data. The expectation for the event numbers at the reconstructed level (denoted as vector $\mathrm{N}$ ) are therefore given as
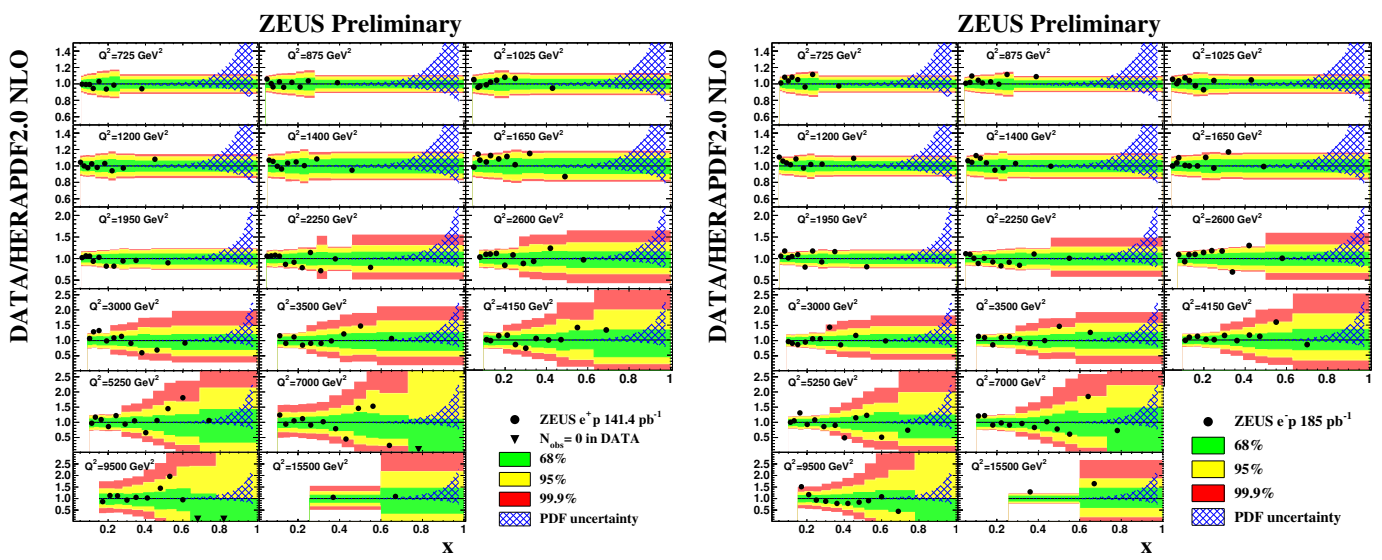

Figure 3: The ratio of the number of observed events to the expectations from the HERAPDF2.0 for $\mathrm{e}^{+} \mathrm{p}$ (left) and $\mathrm{e}^{-} \mathrm{p}$ (right) data respectively. The green, yellow and red bands give the smallest intervals containing $68,95,99 \%$ probability calculated using Poisson statistics.

\section{Results}

Figure 2 shows the comparison of ratio of number of events at the generator level from various modern PDFs $[9,10,11,12]$ to HERAPDF2.0 [1] for $e^{+} p$ (top plot) and $e^{-} p$ (bottom plot) data sets. The PDF uncertainty from HERAPDF2.0 and from NNPDF3.1 [12] is shown in the filled area and hatched area respectively in the given figures. It is observed that the PDFs differ from each other and from HERAPDF2.0 significantly. The difference increases to about $10 \%$ at value of $\mathrm{x}$ of 0.4. At highest values of $x$ the differences increase and the PDF uncertainty also increases.

Figure 3(left) and 3(right) show the statistical comparison of data to the predictions from HERAPDF2.0 using the Poisson statistics for $e^{-} p$ and $e^{+} p$ data sets respectively. The yellow, green and red bands are the smallest intervals [13] conatining 68\%, 95\% and 99\% probabilities for HERAPDF2.0 expectations given the data. The agreement between data and expectations obtained using different PDFs can also be estimated from the p-values [14], which are given in Table 1. It is intriguing to note that for $e^{+} p$ data HERAPDF2.0 is in best agreement with data as compared to the different PDFs, where as for the $e^{-} p$ data other PDFs have a higher $p$-value than the HERAPDF2.0. Table 2 shows the $\mathrm{p}$-values for two different ranges (i.e. $\mathrm{x} \leq 0.6$ and $\mathrm{x} \geq 0.6$ ). It is observed that 
for the higher-x range the PDFs give a similar p-value, whereas for lower-x range the p-values are significantly different.

\begin{tabular}{|c|c|c|}
\hline PDF & $\mathrm{e}^{-} \mathrm{p}$ & $\mathrm{e}^{+} \mathrm{p}$ \\
\hline HERAPDF 2.0 & 0.05 & 0.5 \\
CT 14 & 0.002 & 0.8 \\
MMHT2014 & 0.002 & 0.8 \\
NNPDF 2.3 & 0.00007 & 0.6 \\
NNPDF3.0 & 0.0002 & 0.7 \\
ABMP16 & 0.01 & 0.8 \\
ABM 11 & 0.001 & 0.6 \\
\hline
\end{tabular}

Table 1: $p$-values for full Bjorken-x range for different PDFs (at NLO)

\begin{tabular}{|c|c|c|c|c|}
\hline & \multicolumn{2}{|c|}{$\mathrm{e}^{-} \mathrm{p}$} & \multicolumn{2}{|c|}{$\mathrm{e}^{+} \mathrm{p}$} \\
\hline$P D F$ & $x<0.6$ & $x \geq 0.6$ & $x<0.6$ & $x \geq 0.6$ \\
\hline HERAPDF2.0 & 0.06 & 0.2 & 0.6 & 0.1 \\
\hline CT14 & 0.0008 & 0.2 & 0.7 & 0.6 \\
\hline МMHТ2014 & 0.00003 & 0.1 & 0.6 & 0.6 \\
\hline NNPDF2.3 & 0.00007 & 0.2 & 0.6 & 0.6 \\
\hline NNPDF3.0 & 0.00003 & 0.2 & 0.6 & 0.6 \\
\hline ABMP16 & 0.01 & 0.2 & 0.8 & 0.5 \\
\hline ABM11 & 0.03 & 0.3 & 0.7 & 0.4 \\
\hline
\end{tabular}

Table 2: $\mathrm{p}$-values for two different $\mathrm{x}$ ranges

\section{Conclusions}

In this paper, discriminating power of ZEUS high $\mathrm{x}$ data at the highest Bjorken $\mathrm{x}$ range is shown. The technique to predict the expected number of events from different PDFs using the Transfer matrix for the ZEUS high $\mathrm{x}$ data is discussed. The ZEUS high $\mathrm{x}$ data has some overlap with the ZEUS high $Q^{2}$ data [15] which is already included in the PDF fits, but this data has an extended $\mathrm{x}$-range touching the highest value of $\mathrm{x}$ upto 1 , which could prove to be very helpful in further constraining the PDF uncertainties at this range.

\section{References}

[1] H1 and ZEUS Coll., H. Abramovicz et al., Combinations of measurement of inclusive deep inelastic $e^{+-}$scattering cross sections and qcd analysis of hera data, Eur. Phys. J. C 75, 580 (2015). arXiv:1506.0604

[2] V. N. Gribov and L. N. Lipatov, Deep inelastic e p scattering in perturbation theory, Sov. J. Nucl. Phys. 15, 438 (1972).

[3] V. N. Gribov and L. N. Lipatov, $e^{+} e^{-}$pair annihilation and deep inelastic e p scattering in perturbation theory, Sov. J. Nucl. Phys. 15, 675 (1972).

[4] L. N. Lipatov, The parton model and perturbation theory, Sov. J. Nucl. Phys. 20, 94 (1975).

[5] Yu. L. Dokshitzer, Calculation of the structure functions for deep inelastic scattering and e+eannihilation by perturbation theory in quantum chromodynamics (in russian), Sov. Phys. JETP 46, 641 (1977).

[6] G. Altarelli, and G. Parisi, Asymptotic freedom in parton language, Nucl. Phys. B 126, 298 (1977).

[7] ZEUS Coll., H. Abramowicz et al., Phys. Rev. D 89, 072007 (2014).

[8] A. Kwiatkowski, H. Spiesberger and H.-J. Möhring, heracles: An event generator for ep interactions at HERA energies including radiative processes, CPC 69, 155 (1992). Also in Proc. Workshop Physics at HERA, eds. W. Buchmüller and G.Ingelman, (DESY, Hamburg, 1991).

[9] L. Harland-Lang, A. D. Martin, P. Motylinski and R. Thorne (2014), [arXiv:1412.3989].

[10] M. Guzzi et al., Phys. Rev. D 82, 074024 (2010), [axXiv:1007.2241]. 
[11] S. Alekhin et al., Phys. Rev. D 86, 054009 (2012), [axXiv:1202.2281].

[12] R. D. Ball et al. [NNPDF Collaboration], JHEP 1004, 040 (2015), [arXiv:1410.8849v2]

[13] R. Aggarwal, A. Caldwell, Eur. Phys. J. Plus 127 1-8 (2012).

[14] F. Beaujean et al., Phys. Rev. D 83, 012004 (2011).

[15] ZEUS Coll., H. Abramowicz et al., Measurement of high- $Q^{2}$ neutral current deep inelastic $e^{+} p$ scattering cross sections with a longitudinally polarized positron beam at HERA, Phys. Rev. D 87, 052014 (2013). doi:10.1103/PhysRevD.87.052014. arXiv:1208.6138.

[16] H1 Collab., F. D. Aaron et al., Inclusive Deep Inelastic Scattering at High $Q^{2}$ with Longitudinally Polarised Lepton Beams at HERA, JHEP 1209, 061 (2012). doi:10.1007/JHEP09(2012)061. arXiv:1206.7007. 